\title{
Towards Zero-Waste Furniture Design
}

\author{
Bongjin Koo* \\ University College London
}
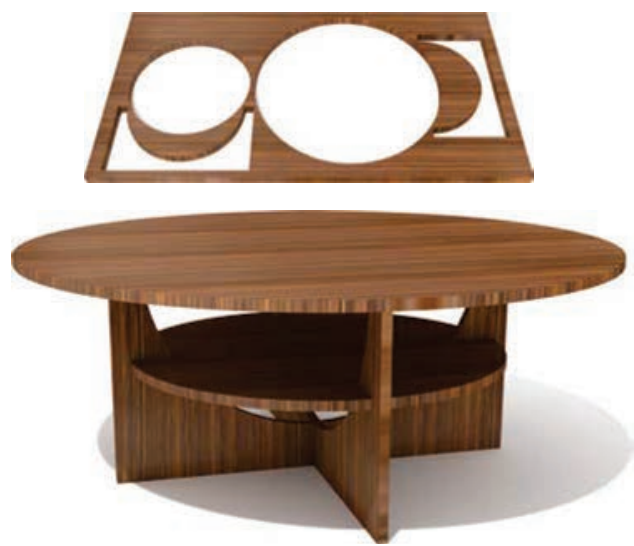

(a) input design

\author{
Jean Hergel* \\ INRIA Nancy
}

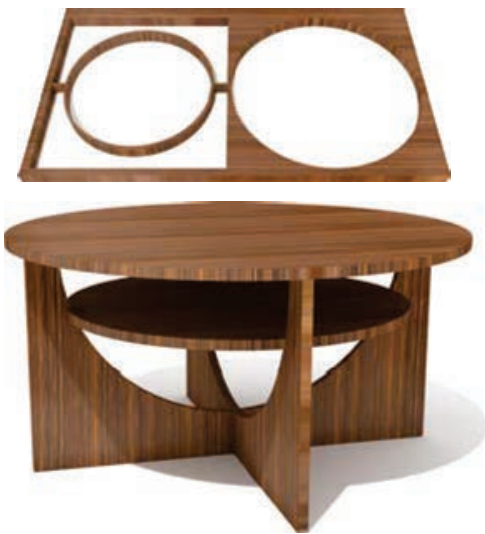

(b) final design
Niloy J. Mitra

University College London
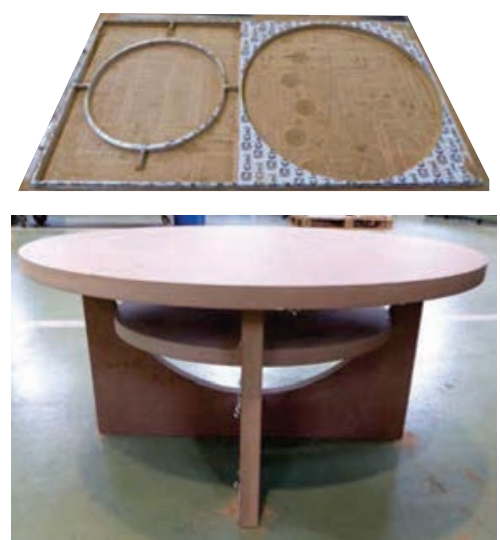

(c) fabricated design

Figure 1: We introduce waste-minimizing furniture design to dynamically analyze an input design (a) based on its $2 D$ material usage (see inset) and design specifications to assist the user through $(b)$ multiple design suggestions to reduce material wastage (see inset). The final user design can directly be exported for laser cutting and be assembled (c). In this case, wastage was reduced from $22 \%$ to $11 \%$.

\section{Abstract}

In traditional design, shapes are first conceived, and then fabricated. While this decoupling simplifies the design process, it can result in inefficient material usage, especially where off-cut pieces are hard to reuse. The designer, in absence of explicit feedback on material usage remains helpless to effectively adapt the design - even though design variabilities exist. We investigate waste minimizing furniture design wherein based on the current design, the user is presented with design variations that result in more effective usage of materials. Technically, we dynamically analyze material space layout to determine which parts to change and how, while maintaining original design intent specified in the form of design constraints. We evaluate the approach on simple and complex furniture design scenarios, and demonstrate effective material usage that is difficult, if not impossible, to achieve without computational support.

Keywords: computational design, fabrication, material usage, guided design

\section{Introduction}

Furniture design is an exercise in form-finding wherein the designer arrives at a final form by balancing aesthetics, object function, and cost. Typically, design variations are manually explored by a mixture of guesswork, prior experience, and domain knowledge. Without appropriate computational support, such an exploration is often tedious, time consuming, and can result in wasteful choices.

In furniture manufacturing, both for mass production and for customized designs, material wastage plays a deterrent role. This not only leads to increased production cost (typically 5-15\% wastage due to off-cuts), but also hampers ongoing efforts towards green manufacturing [Daian and Ozarska 2009]. For an extensive report, please refer to the guideline from the British Furniture Manufacturer [BFM 2003]. Hence, there has been a growing interest in

* Joint first authors zero-waste furniture in an effort to reduce material wastage. A notable example being Maynard's 'Zero-waste Table.' Computational support for designing such waste-reducing furniture, however, is largely lacking.

Material considerations are typically appraised only after a shape has been designed. While this simplifies designing, it leads to unnecessary wastage: at design time, the user can at best guess to account for how the shape will be physically realized, and can easily fail to effectively adjust the design to improve material utilization.

In recent years, algorithms have been developed to economically $3 \mathrm{D}$ print given designs. For example, approaches have been proposed to cleverly breakup a given shape into parts that better pack together in print volumes [Luo et al. 2012; Vanek et al. 2014; Chen et al. 2015; Yao et al. 2015], adaptively hollow shape interiors to save print materials [Stava et al. 2012; Prévost et al. 2013; Wang et al. 2013; Dumas et al. 2014], explore parameter space variations for manufacturable forms [Shugrina et al. 2015], or design connector geometry to remove the need for any secondary connector parts [Fu et al. 2015]. However, improving material utilization by explicitly allowing design changes has been less studied.

In this work, we introduce the problem of waste-minimizing furniture design, and investigate it in the context of flatpack furniture design (cf., [Brennan et al. 2006]) using laser cut wooden parts. Specifically, we study the interplay between furniture design exploration and cost-effective material usage. By directly coupling the two, we empower the users to make more informed design decisions. Note that this is fundamentally different from locking a designed shape, and then trying to best fabricate it.

For example, in Figure 1, the user starts with an initial concept indicating design constraints (e.g., symmetry, desired height, etc.). Our system analyzes material usage by computing a dynamic 2D layout of the parts and proposes design modifications to improve material usage without violating specified design constraints (i.e., design intent). Note that such adaptations are often in the form of synchronous movement of multiple parts affected by both design 
and material layout considerations, which are difficult to mentally imagine. The user can select any of the suggestions, either in its entirety or in part. She can further update the set of design constraints by locking parts of the current design, and the process continues. Thus, the user scopes out a design space via constraints, and our algorithm refines the design to reduce material wastage while restricting changes to the indicated design space.

Technically, we achieve the above by using the current material layout to dynamically discover a set of relevant layout constraints. The algorithm has a discrete aspect involving which part to change based on the current 2D layout, and a continuous aspect involving how to adapt the part attributes based on the current material space layout without violating user-specified design constraints. Even for a fixed design, exploring the space of all possible packing is a combinatorial NP-hard problem. Instead, we locally analyze a set of candidate packings to determine which parts to modify and how to change them to optimize material utilization. We demonstrate that by dynamically analyzing a set of current packings, we can efficiently and effectively couple the 2D layouts and the constrained 3D designs. The user is then presented with different waste-reducing design variations.

We evaluated the system to create a variety of simple and complex designs, and fabricated a selection of them. We also performed a user study with both designers and novices to evaluate the effectiveness of the system. The performance benefits were particularly obvious in case of complex designs involving different design constraints. In summary, we:

- introduce the problem of material waste minimizing furniture design; and

- propose an algorithm that dynamically analyzes 2D material usage to suggest design modifications to improve material usage without violating user-specified constraints.

\section{Related Work}

Material considerations. Physical materials play an important role in 3D printing an object. Various approaches have been developed to economically and efficiently produce a designed object. For example, adaptively hollowing out interiors and adding struts to create durable yet cost-effective 3D printouts [Stava et al. 2012], cleverly hollowing the shape interiors in conjunction with shape deformation to ensure stability of the final shape [Prévost et al. 2013], or perform FEM analysis to decide wall thickness and parameters to ensure model endurance under known or unknown forces [Zhou et al. 2013; Lu et al. 2014]. Techniques for designing scaffolds, both interior [Wang et al. 2013] and exterior [Dumas et al. 2014], have been developed for cost-effective $3 \mathrm{D}$ printing by reducing wastage. Hu et al. [2015] propose to optimize the shape of a 3D model to reduce support structures used during 3D printing. Alternatively, methods have been developed to decompose and pack 3D models for reducing assembly cost, support material, printing time or making big objects printable on small 3D printers [Luo et al. 2012; Vanek et al. 2014; Yao et al. 2015]. Dapper [Chen et al. 2015] also employs a decompose-and-pack approach for minimum assembly cost, support material and build time when using $3 \mathrm{D}$ printers. It breaks $3 \mathrm{D}$ objects into pyramidal primitives, then finds good packing configurations to achieve the goal.

In the context of laser cut fabrication, Hildebrand et al. [2012] and Schwartzburg and Pauly [2013] explore how to rationalize a given design for fabrication out of planar sheets. Further, material wastage has been investigated by testing various packing strategies from computational geometry community (cf., [Jylänki 2010]) to efficiently layout the parts in the material space. More recently,
Saakes et al. [2013] proposed an interactive system to allow the user to interactively layout parts for more personalized usage. Such methods, however, do not explicitly modify the original designs in order to improve material usage.

Fabrication-aware design. Recently, the growing popularity of personalized fabrication has motivated researchers to develop algorithms to adapt existing shapes to make them better suited for physical construction. Examples include abstracting shapes as a collection of slices to be laser cut [McCrae et al. 2011; Hildebrand et al. 2012; Schwartzburg and Pauly 2013; Cignoni et al. 2014], as foldable popups [Li et al. 2010; Li et al. 2011], developing toolkit to allow user to draft directly using a handheld laser pointer to control high-powered laser cutters [Mueller et al. 2012], computationally designing gear trains to support part movement for converting animated characters to working physical automata [Coros et al. 2013], introducing necessary joint geometry to create non-assembly articulated objects [Bächer et al. 2012; Calì et al. 2012], or supporting an example-driven fabrication paradigm [Schulz et al. 2014]. To simplify fabrication, Fu et al. [2015] suggest a method to generate a globally-interlocking furniture assembly that enables easy disassembly/reassembly of furniture, without using glue, screws, etc. Such methods, however, are chiefly used to adapt existing shapes after they have been designed, rather than to guide the user to refine the designs to reduce material-wastage.

Guided design. In the context of exploratory design, $\mathrm{Xu}$ et al. [2012] proposed a fit-and-diverse framework to allow users to interactively guide model synthesis and exploration, while Talton et al. [2009] exposed a parameterized shape space for model creation. These efforts, however, focus on aspects of digital content creation without fabrication and material considerations. Recently, Shugrina et al. [2015] developed a system that allows novices to easily customize parametric models while maintaining 3D-printability of the models. In a work closely related to our motivation, Umetani et al. [2012] use stability and durability of materials to propose design modifications, thus computationally guiding the users. With a similar motivation, we investigate the impact of material usage in the context of guided design. We are unaware of prior attempts investigating how material usage can be analyzed to refine the designs.

Constraint-based modeling. In the CAD community, constrainedbased modeling (cf., [Brüderlin and Roller 1998]) has long been demonstrated as a powerful parametric way to design shapes and interact with them. In the case of existing models, an inverse analyzeand-edit paradigm has been recently proposed to first discover the constraints present in shapes, and then allow interactive editing [Gal et al. 2009; Xu et al. 2009; Zheng et al. 2011]. Such approaches differ on how model parts are abstracted (e.g., feature curves, model parts, or abstracted segments as primitives) and how the inter-part constraints are conformed to. However, these methods have primarily focused on designing shapes for the virtual world where material and fabrication constraints are irrelevant, and hence ignored.

\section{Design Workflow}

Our goal is to propose design variations that minimize material wastage without violating original design intent. In this section, we present the proposed system as experienced by the user, and describe the main algorithmic details in the subsequent sections. Here we particularly focus on how the user encodes her design intent.

The user starts by choosing the desired material (i.e., thickness of wooden planks) and the number and dimensions of the master board(s). Our system considers rectangular master boards in practice these can represent new boards or left over rectangular 
spaces in already used boards. The user starts by loading an initial part-based 3D object design, either created in a modeling system or as a parameteric model. The parts can be rectangular or have curves boundaries. The user also indicates a set of design constraints. In our implementation, we support: equal length (e.g., $l_{i}=l_{j}$ ), sum of lengths (e.g., $l_{i}+l_{j}+\cdots=l_{k}+\ldots$ ), fixed length (e.g., $l_{i}=c$ ), equal position, symmetric parts, ground touching, and coplanarity among indicated planks. The user can additionally specify that the object should fit an indicated volume (e.g., in between two walls) and the internal space in the form of inner volume indicating minimal shelf dimensions.

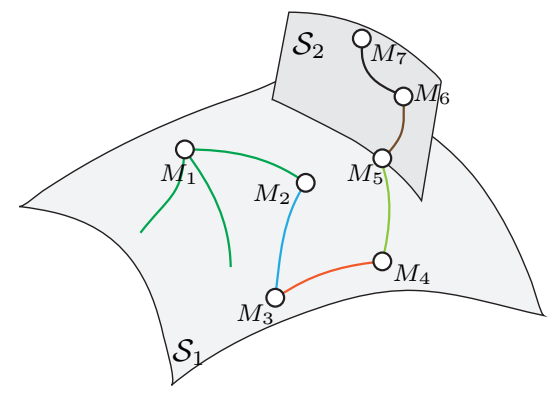

Figure 2: Our algorithm discovers design variations in shape space. The user starts from a design $M_{1}$ along with indicated design constraints, and the algorithm seeks for wastage minimizing variations by interleaving between topologically different material layouts (indicated by changes in curved paths) or continuous changes to the layouts (indicated by same colored curves). For example, paths $\left(M_{i}, M_{j}\right)$ denote continuous design changes, while points $M_{i}$ denotes designs where new layouts are explored (i.e., branch points). The user can switch to another shape space by picking an updated set of design constraints (shape $M_{5}$ here). Note that by construction $M_{5}$ belongs to both shape spaces $\mathcal{S}_{1}$ and $\mathcal{S}_{2}$. See Algorithm 1.

The algorithm suggests multiple design variations that all satisfy the design specifications but achieve different material usages. We measure material usage based on the fraction of the master board(s) utilized. The top suggestions are presented as thumbnails. If the user mouse-overs any thumbnail, the system animates the proposed design modifications. The user can preview the object- and material-space views, and select her preferred design suggestion. Note that each thumbnail effectively represents a design exploration path pursued by the algorithm. We provide a slider to move along this path, which is particularly useful for making incremental updates to the design (see Figure 2 and Section 4).

The user either selects a suggested design variation, or picks part configurations from a suggested shape as additional design constraints (e.g., user can lock the proposed sizes of certain planks). Thus, effectively the user appends or updates the current set of specified design. Note that the new constraints are trivially satisfied by the current design, which is critical for subsequent design space exploration (e.g., $M_{5}$ is in both shape spaces $\mathcal{S}_{1}$ and $\mathcal{S}_{2}$ ).

Once satisfied with a design, she requests for the cutting patterns. She can investigate the design, the material space usage and the cutting patterns, and send the patterns directly for laser cutting.

\section{Overview}

Our goal is to analyze aspects arising from material considerations, and investigate how design changes affect such considerations. Specifically, we ask how to adapt a furniture design so that it makes better utilization of material in the resultant design layout. Note that this is the inverse of the design rationalization problem, i.e., instead of taking a design as fixed and best fabricating it, we adapt the design so that the resultant rationalization makes better utilization of available material. First, we introduce some notations.

\subsection{Parameterized designs}

The design is considered as a function $D(\mathbf{X})$ that produces the geometry of a fixed number of parts, given a configuration vector $\mathbf{X}$. The parts can be assembled into a final furniture design.

We make no assumption as to how $D$ is implemented - we demonstrate in Section 6 applications using both constrained based furniture design and parametric designs modeled by CSG. We however expect a continuous behavior from $D(\mathbf{X})$, i.e., small changes in $\mathbf{X}$ result in small changes in the part shapes. Parametric modelers generally offer such continuity to smoothly navigate the space shape.

During wastage optimization our algorithm will change the value of $\mathbf{X}$ so as to explore whether changes in part shapes reduce wastage. Since we focus on laser cut furniture construction, we assume the parts to have the same thickness $\tau$. The parts are thus represented as planar polygonal contours extruded orthogonally.

The geometry of a part $p_{i}$ lies within a bounding box which we represent by a six dimensional vector encoding the box center $\mathbf{p}_{i}$ and the lengths of its three sides $l_{i}^{x}, l_{i}^{y}, \tau-$ the $\mathrm{Z}$ axis being aligned with part thickness by convention.

\subsection{Material space}

Since we focus on laser cut furniture, any 3D design given by a configuration vector $\mathbf{X}$ is realized as a layout (i.e., cutting plan) in the material space. Material space is characterized by the largest master board that the machine can possibly cut, a rectangle of size $W \times H$. In this space, each part $i$ is associated with a position $\left(u_{i}, v_{i}\right)$ and an orientation $o_{i} \in\{0, \pi / 2, \pi,-\pi / 2\}$.

We use $w_{i}, h_{i}$ as extent of a part bounding box in the material space along the $\mathrm{x}$ - and $\mathrm{y}$-axis, respectively. The part box lengths in material space are given by the two plank dimensions other than thickness. For a plank $i$, of orientation $o_{i}$, we get one of the two cases:

$$
\begin{array}{llll}
o_{i}=0, o_{i}=\pi & \Rightarrow & w_{i}=l_{i}^{x} & h_{i}=l_{i}^{y} \\
o_{i}=-\pi / 2, o_{i}=\pi / 2 & \Rightarrow & w_{i}=l_{i}^{y} & h_{i}=l_{i}^{x}
\end{array}
$$

The material space positions and orientations are variables in the layout optimization algorithm, alongside the design parameters $\mathbf{X}$ (see Section 5).

When wastage is not a concern and a design easily fits within material space, the variables $\left(u_{i}, v_{i}, o_{i}\right)$ are independent of the design, i.e., they simply adapt to changes in part sizes. However, as we seek to maximize utilization of the material space, the material space variables become tightly coupled with the design parameters. Our layout optimizer therefore jointly optimizes for material space variables and design parameters to minimize wastage (see Section 5)

We next discuss what makes a desirable layout from the point of view of furniture fabrication.

\subsection{Properties of a good design layout}

Rectangular master boards can be sourced in a large choice of sizes and thicknesses from resellers. Therefore, our goal is to achieve a full utilization of rectangular spaces, so that the user can use boards 

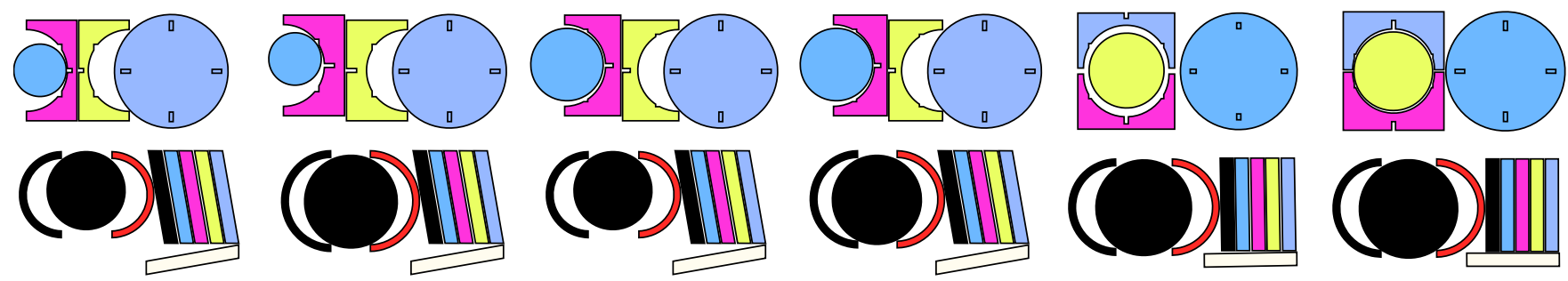

Figure 3: Evolution of shape variation across a run of our algorithm on the coffee-table (top) and low-chair (bottom) models.

of exactly the right size and minimize wastage. The machine dimensions determine the maximum extent of a single board.

We measure wastage as the fraction of the space not utilized by the design in its material space bounding rectangle. Ideally, we want to achieve full utilization, i.e., null wastage.

An ideal packing is one that tightly packs all the parts to perfectly fill up one or more rectangular master boards (like a puzzle). Our system helps the user achieve this by automatically exploring changes improving material space usage (see Figure 4).

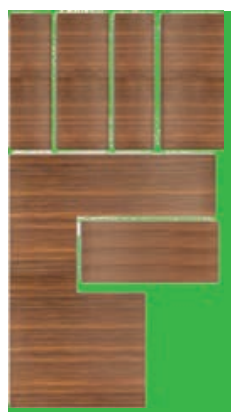

bad layout

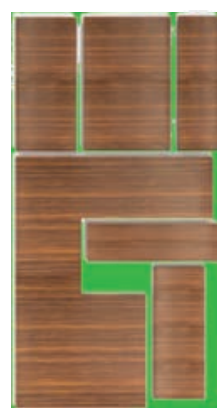

mediocre layout

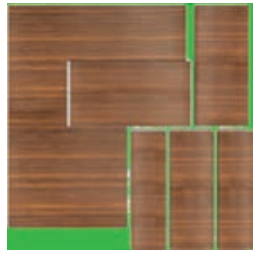

good layout
Figure 4: Examples of stages of layout refinement, from bad to mediocre to good. A good layout is characterized by less area of material wasted (shown in green).

\section{Design Layout Optimization}

The wastage of a layout depends essentially on two factors. The first factor is the quality of the packing that can be achieved, given

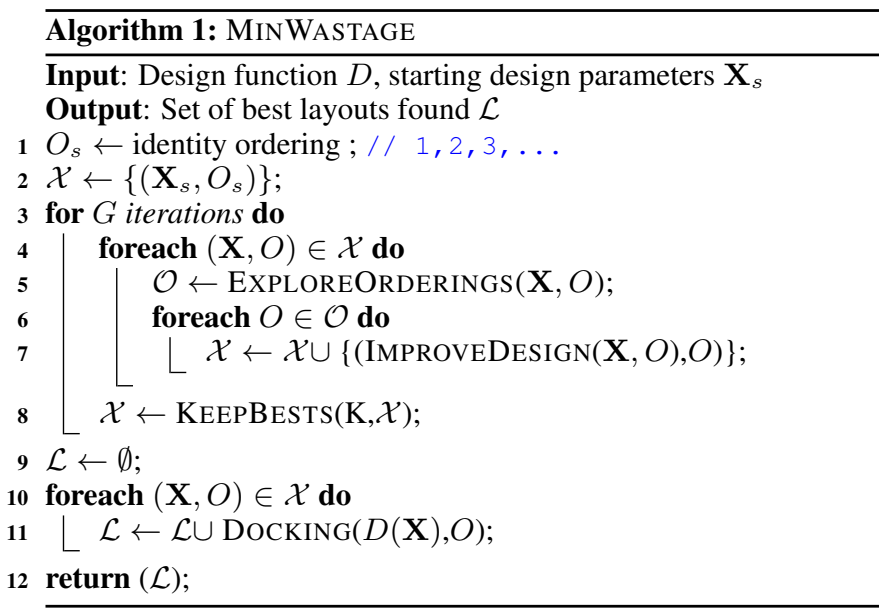

a fixed set of design parts. The second factor is the set of parts itself, which can be changed through the design parameters $\mathbf{X}$.

In our approach we pack the parts using a deterministic docking algorithm that always produces the same result for a same ordering of the design parts. Therefore, a first optimization variable is the order in which the parts are sent to the docking algorithm. The second optimization variable is the vector of design parameters $\mathbf{X}$. These two variables have different natures: finding an ordering is a combinatorial problem while the design parameters can be continuously explored.

We therefore proceed in two main steps, first determining a set of good orderings that then serve as starting points for continuously evolving the design, reducing wastage. The overall approach is described in Algorithm 1. The subroutine IMPROVEDESIGN is described in Section 5.1 while EXPLOREORDERINGS is described in Section 5.2. The process restarts for a number of iterations (we use $G=3$ ) to jump out of local minima reached by the continuous design exploration. This results in the shape space exploration illustrated in Figure 2. The process returns the $K$ best found layouts and designs and presents them to the user in thumbnails. She can then select her favorite design, and if desired update the constraints and restart the exploration from this point — which simply calls MinWastage again.

Bitmaps. During optimization we regularly call the parameterized design function $D(\mathbf{X})$ to obtain a new set of parts after changing parameters. The layout optimization represents parts internally as bitmaps: each part contour is rasterized at a resolution $\tau$, typically $0.5 \mathrm{~mm}$ per pixel. This enables fast manipulation of the parts within the layout. Each part thus becomes a bitmap having either 1 (inside) or 0 (outside) in each pixel. The size of the bitmap matches the part extents in material space $w_{i}$ and $h_{i}$. Every time the design is refreshed a new set of bitmaps is computed for the parts. The master board is similarly discretized into a regular grid of resolution $\tau$.

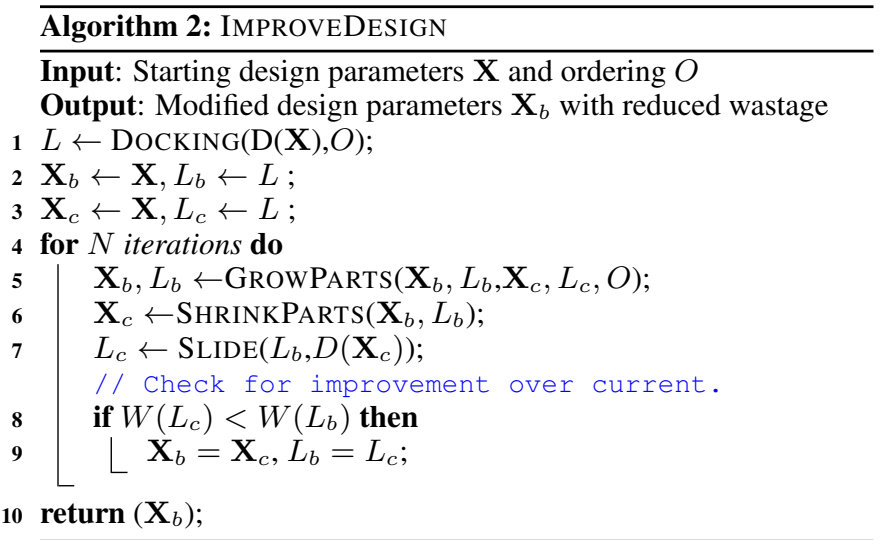




\subsection{Design optimization for wastage minimization}

The design optimization improves the design parameters $\mathbf{X}$ to minimize wastage in the layout, keeping the docking ordering fixed. It appears as the subroutine IMPROVEDESIGN in Algorithm 1. The pseudo-code for this step is given in Algorithm 2. Our objective is to suggest design changes that reduce wastage, progressively improving the initial layout. The algorithm performs a guided local search by changing the parts - through the design parameters - to reduce wastage.

Prior to considering which parts to modify, we have to answer two questions: First, how to drive the design parameters $\mathbf{X}$ to change only a given part (Section 5.1.1). This is achieved by relying on the gradients of the part size with respect to $\mathbf{X}$. Second, we have to decide on how to evolve the layout when parts are changed (Section 5.1.2). We rely on a sliding algorithm that avoids jumps in the layout configuration, thus producing only small changes in the wastage function when small changes are applied to the part sizes.

Overall strategy. Our approach changes the size of parts iteratively with two different steps in each iteration: grow (line 5) and shrink (line 6). These steps progressively modify the design and keep track of the design of smallest wastage encountered so far.

The grow step (Section 5.1.3) attempts to enlarge the parts so as to reduce wastage. Each part is considered and its size is increased for as long as the growth further reduces wastage. When no further improvement can be obtained, we create further opportunities by shrinking a set of parts (Section 5.1.4). However, randomly shrinking parts would be inefficient, as most parts would grow back immediately to their original sizes. Other parts are tightly coupled to many others in the design $D$, and shrinking these would impact the entire design. Therefore, we analyze the layout to determine which parts have a higher probability to result in wastage reduction.

\subsubsection{Changing part sizes}

During design space exploration the algorithm attempts to vary the part sizes $w_{i}$ and $h_{i}$ individually. These dimensions vary as a function of design parameters $\mathbf{X}$. In the remainder we use $\mathbf{s}(\mathbf{X})$ to designate the vector of all part sizes assembled such that $s_{2 i}=w_{i}$ and $s_{2 i+1}=h_{i}$.

Let us denote $\lambda$ the change of size desired on $s_{i}$. Our objective is to compute a design change $\Delta$ such that $s_{i}(\mathbf{X}+\Delta)=s_{i}(\mathbf{X})+$ $\lambda$. We denote the vector of changes as $\Lambda=\mathbf{s}(\mathbf{X}+\Delta)-\mathbf{s}(\mathbf{X})$. In this process only the size $s_{i}$ should change with others remain unchanged whenever possible, that is $\Lambda_{s_{j}, j \neq i}=0$ and $\Lambda_{s_{i}}=\lambda$.

Parts are not independent in the design and therefore there is no trivial link between $\mathbf{X}$ and $s_{i}(\mathbf{X})$. We therefore analyze the relationship through the gradients $\frac{\partial s_{i}(\mathbf{X})}{\partial x_{j}}$. These are computed by local finite differencing (depending on the design analytical expressions may be available). Each non-null gradient indicates that parameter $x_{j}$ influences $s_{i}$. Multiple parameters may influence $s_{i}$ and parameters typically also influence other variables: there exists $k \neq i$ such that $\frac{\partial s_{k}(\mathbf{X})}{\partial x_{j}} \neq 0$.

To compute $\Delta$ we formulate the following problem. Let us consider the components of $\Delta=\left(\delta_{0}, \ldots, \delta_{|\mathbf{X}|-1}\right)$. The change in part sizes due to $\Delta$ can be approximated in the first order through the gradients as $\boldsymbol{\Lambda}=\sum_{i} \delta_{i} \cdot \frac{\partial \mathbf{s}(\mathbf{X})}{\partial x_{i}}$. We solve for $\Delta$ such that $\Lambda_{s_{i}}=\lambda$ and $\Lambda_{s_{j}, j \neq i}=0$.

If there are less parameters than part sizes, the problem is overconstrained and solved in the least-square sense, minimizing $\| \boldsymbol{\Lambda}-$ $(0, \ldots, \lambda, \ldots, 0) \|^{2}$. If there are more parameters than part sizes, the

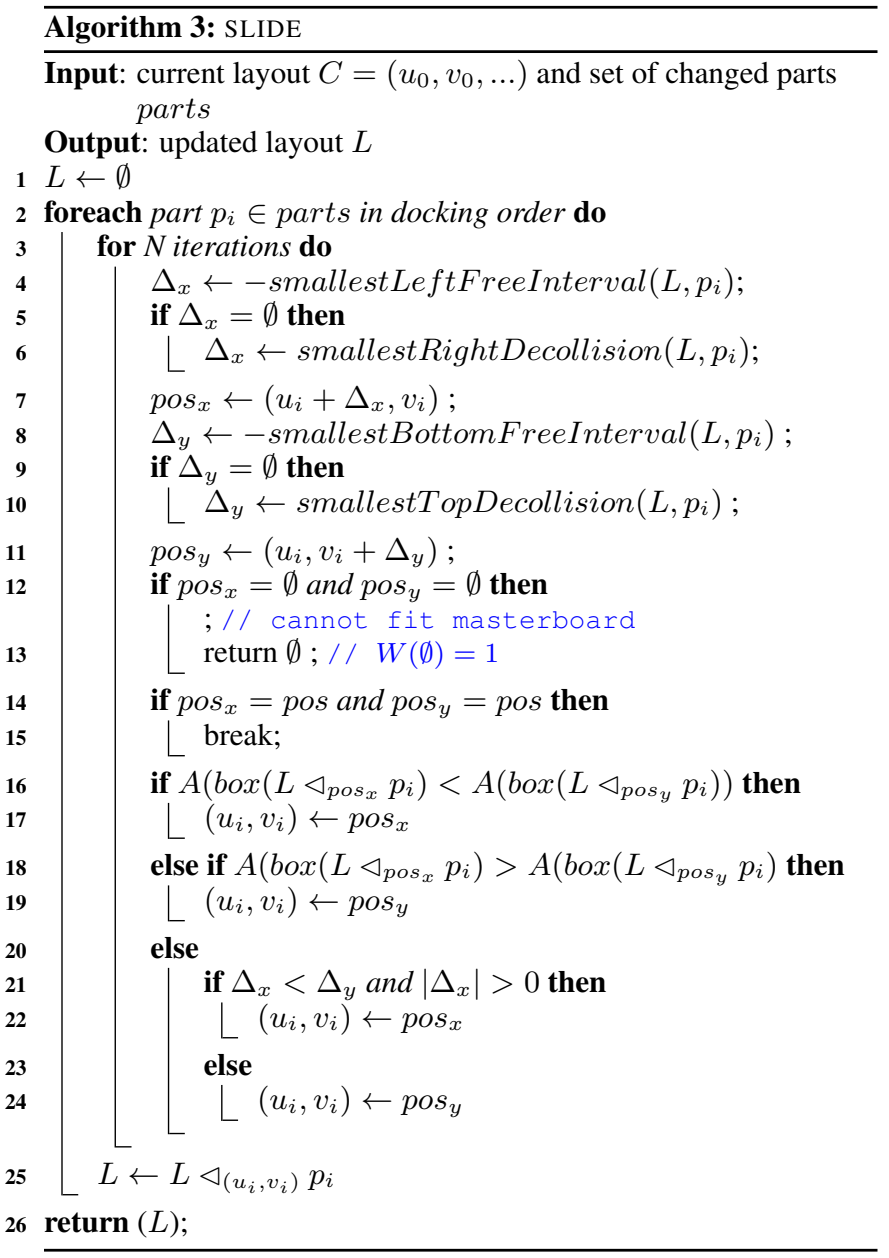

problem is under-constrained and solved in the least-norm sense, minimizing $\|\Delta\|$. We rely on a QR decomposition of the system matrix to solve for both cases, accounting for possible rank deficiencies due to overlapping parameters in $\mathbf{X}$.

We implement this process as a subroutine ChangePartSize $\left(\mathbf{X}, s_{i}, \lambda\right)$, with $\mathbf{X}$ the current design parameters, $s_{i}$ the part size to change and $\lambda$ the change to apply. It returns the new design parameters $\mathbf{X}+\Delta$. A second subroutine ChangePartSizes $(\mathbf{X}, \Lambda)$ allows to change the size of multiple parts at once.

\subsubsection{Updating layouts by sliding}

As the shapes and sizes of the parts change the layout has to be updated. One option would be to restart the docking process after each change. However, for a small change the docking process can produce large discontinuities in the wastage function. This makes a local search difficult. Instead, we propose to rely on a sliding operation that attempts to continuously update the position of the parts after each change. Note that performing such an update while optimizing for a given objective (i.e. wastage) is a very challenging combinatorial problem, as each part can move in four directions (left/right/top/bottom) and multiple cascading overlaps have to be resolved. We propose a heuristic approach that works well for small changes in the part shapes.

The algorithm is based on the following principle. After changing the part shapes, we reintroduce them in an empty layout in order of 
docking. However, each time a part is reintroduced it may now have empty space to its left/bottom or it may overlap with previously placed parts. Both cases can be resolved by a single horizontal or vertical move. However a single move is generally not desirable as empty space may remain along the other direction. We therefore perform a limited sequence of horizontal/vertical moves. At each iteration we select between vertical or horizontal by favoring moves that result in the smallest layout bounding box. In case of a tie, we favor moves to the left/bottom versus displacements to the top/right. This is illustrated in Figure 5.
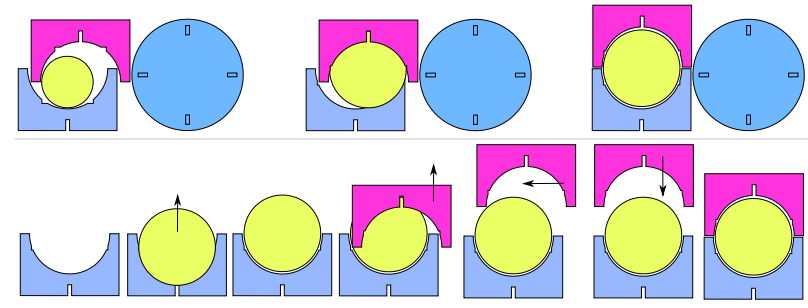

Figure 5: Sliding a layout after a change of part sizes. Top: From left to right, initial layout, same after change revealing overlaps, layout after sliding. Bottom: Moves performed on the three first parts during sliding.

The pseudo-code is given in Algorithm 3. In the algorithm we denote by $L$ the layout and denote by $L \triangleleft_{\text {pos }} p_{i}$ the layout obtained when adding part $p_{i}$ at position pos in the master board grid of $L$. $A($.$) measures the area, \operatorname{box}(L)$ is the bounding rectangle of the layout. The algorithm iterates over all parts in docking order (line 2). It then performs a fixed number of sliding operations on each part (line 3) - we use $N=4$ in our implementation. Lines 4-7 compute a horizontal move, favoring moves to the left that collapse newly created empty spaces. Lines 4-7 similarly compute a vertical move. Lines 16-24 decide whether to select a horizontal move $p_{0} s_{x}$ or vertical move $\operatorname{pos}_{y}$.

The process may fail if parts can no longer fit in the masterboard. This can happen either because there is not enough remaining area, or because sliding cascades in large moves that prevent further insertion of parts. In such cases we return an empty layout which by convention has a wastage of 1 (worst possible), line 13 .

\subsubsection{Grow step}

The grow step is described in Algorithm 4. The algorithm iterates over all parts in random order (line 4) and progressively increases the size of a part in a loop (line 7). Note that the first iteration of the loop determines the starting wastage for growing this part (lines 5 and 12-13). The process continues until the growth results in an increased wastage (line 15).

After each change of parameters the design parts are recomputed (line $9, D\left(\mathbf{X}_{\mathbf{e}}\right)$ ) and sliding is called to adapt the current layout to the change. The result is checked. If wastage decreases the process continues (line 13). If not, we first attempt to dock the parts again (line 11). This can help continue the growth in cases were sliding fails to resolve overlaps by continuous changes. If wastage still not improves we stop the growth of this part size (line 15).

\subsubsection{Shrink step}

The goal of the shrink step is to create further opportunities for design changes when no parts can further grow. The typical situation is that a subset of parts are forming locking chains between respectively the left/right and top/bottom borders. The parts belonging to

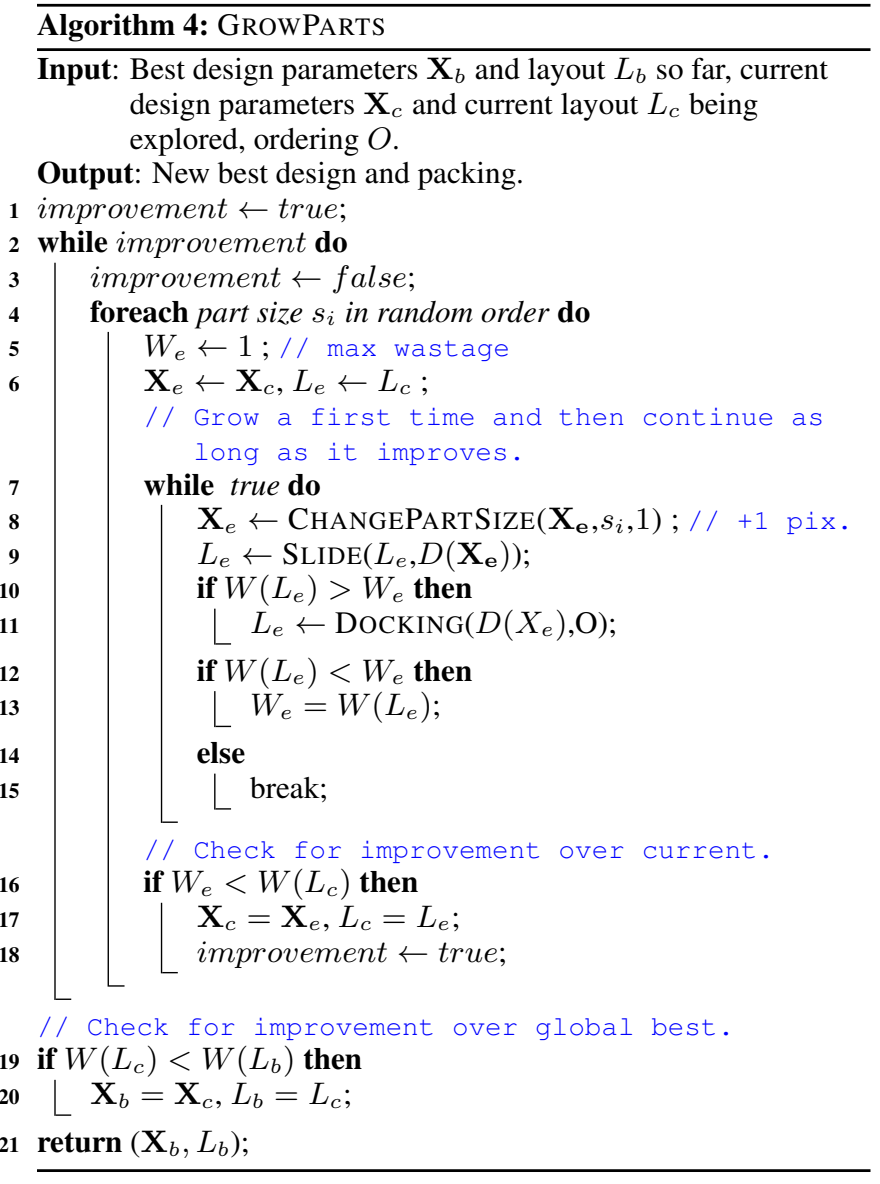

these chains prevent any further growth. We therefore detect locking chains and select the parts to shrink among these. This often results in a change of aspect ratio of the masterboard, and new opportunities for other parts to grow.

The overall approach is described in Algorithm 5. It first determines which parts to shrink by calling SELECTPARTSTOSHRINK and then computes a change of parameters using the approach described in Section 5.1.1.

The core component is the SELECTPARTSIZESTOSHRINK subroutine, described in Algorithm 6. The selection starts by gathering all contacts between parts in the layout - this is done efficiently in the discretized layout grid. We first draw the part images into the grid and then check pairs of neighbors belonging to different parts. This produces the set of left/right and bottom/left contacts between part sizes (the involved part size is deduced from the part

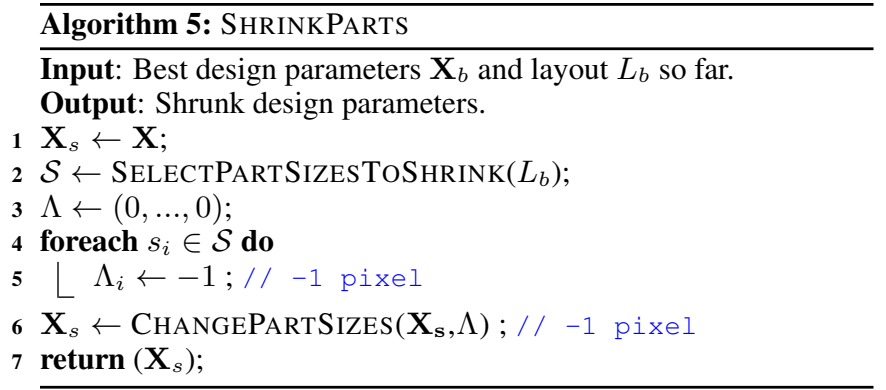




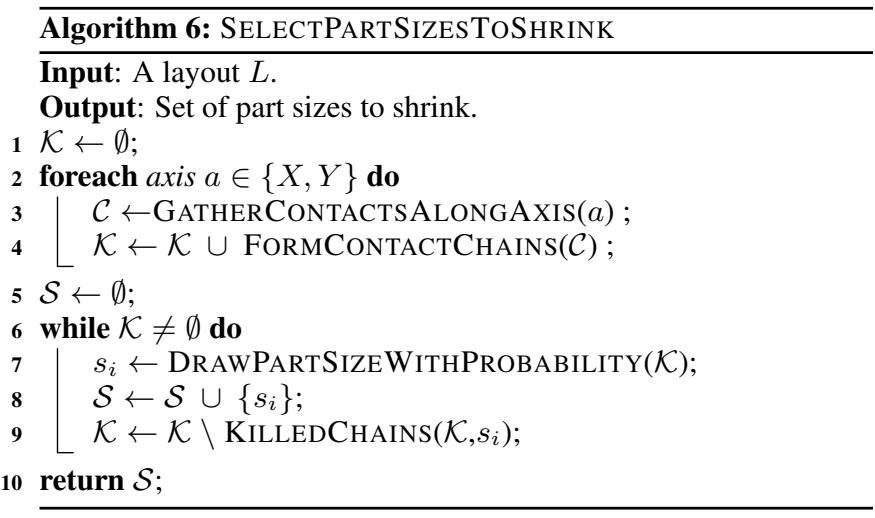

orientation and the considered axis). The contacts are oriented from right to left (respectively top to bottom). We similarly detect which parts touch the borders. The contact detection is implemented in the GATHERCONTACTSALONGAXIS subroutine.

Once the contacts are obtained we start from the right (respectively top) border and form locking chains. Starting from the border, we produce the set of chains iteratively. Each chain $c$ is a sequence $\left(l e f t, s_{\text {first }}, \ldots, s_{\text {last }}\right)$. At each iteration the chain spawns new chains for each contact pair $\left(s_{\text {last }}, s_{\text {next }}\right)$ obtained by augmenting $c$ as $\left(l e f t, s_{\text {first }}, \ldots, s_{\text {last }}, s_{\text {next }}\right)$. Potential cycles are easily detected as repetition of a same part in the chain and are ignored. The locking chain computation is implemented in the FORMCONTACTCHAINS subroutine.

We next randomly select part sizes to shrink until all locking chains are removed. The selection probability of each part is designed to avoid too large a jump in the design space. To achieve this we consider two factors. First, we compute the number of occurrences of each part in the locking chains, $\operatorname{occ}\left(p_{i}\right)$. A part with many occurrences is a good candidate as shrinking it will resolve multiple locking chains at once. Second, we seek to avoid shrinking part sizes that are tightly coupled with others in the design $D$. We compute the dependence of a part size by counting the number of non-zero entries in the $\boldsymbol{\Lambda}$ vector computed internally by ChangePartSize $\left(\mathbf{X}_{\mathbf{e}}, s_{i},-1\right)$.

We select part sizes with the following random process. First, we select a number of occurrences $o$ with probability $P(o)=$ $\frac{\sum_{p_{i}, o c c(p i)=o} o c c(o)}{\sum_{p_{i}} o c c\left(p_{i}\right)}$. Then, among the parts such that $o c c\left(p_{i}\right)=o$ we select a part size $s_{i}$ with probability $P\left(s_{i} \mid \operatorname{occ}\left(s_{i}\right)=o\right)=$ $1-\frac{\operatorname{dep}\left(s_{i}\right)}{\sum_{p_{i}, o c c\left(p_{i}\right)=o} \operatorname{dep}\left(p_{i}\right)}$. This process is implemented by the DRAWPARTSIZEWITHPROBABILITY subroutine.

After each part size selection we update the set of locking chain by removing all chains where the part size appears.

\subsection{Exploring orderings}

The subroutine EXPLOREORDERINGS in Algorithm 1 performs a stochastic search of orderings resulting in low wastage layouts. The process starts from a random order and iteratively considers possible improvements by swapping two parts. At each iteration, we perform a swap and recompute a layout using the docking algorithm. If wastage is reduced the swap is accepted, otherwise it is rejected. We apply the process for a number of iterations and keep the best ordering found as the starting point. We use $|D(\mathbf{X})|^{2}$ iterations, where $|D(\mathbf{X})|$ is the number of parts. For each ordering, we use a fast docking algorithm to compute a layout with low wastage.
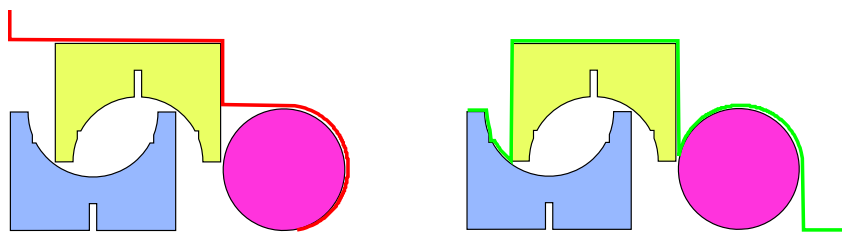

Figure 6: Height-fields of the layout used to position the next part. Left: Height-field for dropping parts from the right (red curve). Right: Height-field for dropping parts from above (green curve). These height-fields are maintained every time a new part is added to the layout, and used for fast computation of the docking positions. Similar height-fields are pre-computed for the left/bottom of the parts.

Docking algorithm. The docking algorithm places each part in order by 'dropping' the next part on the current layout either from the right, or from the top. It locally searches for the best placement of each part, according to a criterion that minimizes wastage. The result is a layout $L$ including all parts.

Given the layout so far our algorithm searches for the best orientation and best position for the next part. We denote by $L_{i-1}$ the layout obtained for the $i-1$ first parts, and by $L_{i} \leftarrow L_{i-1} \triangleleft_{\text {pos }} p_{i}$ the layout obtained by adding the next part at position pos. The docking position pos is computed from a drop location $(s, x, o)$, with $s \in\{$ top, right $\}, x$ a position along the corresponding axis and $o \in\{0, \pi / 2, \pi,-\pi / 2\}$ an orientation.

The pseudo code for the docking algorithm is given in Algorithm 7. The drop locations are ranked according to a docking criterion that we denote $D\left(L_{i-1}, p_{i}, p o s\right)$, explained next. The docking positions are computed from the drop locations by the ComputeDockingPosition subroutine. It is efficiently implemented by maintaining the right/top height-fields of the current layout as illustrated in Figure 6. Whenever evaluating a drop location we use the height-fields to quickly compute the docking positions that bring the part in close contact with the current layout.

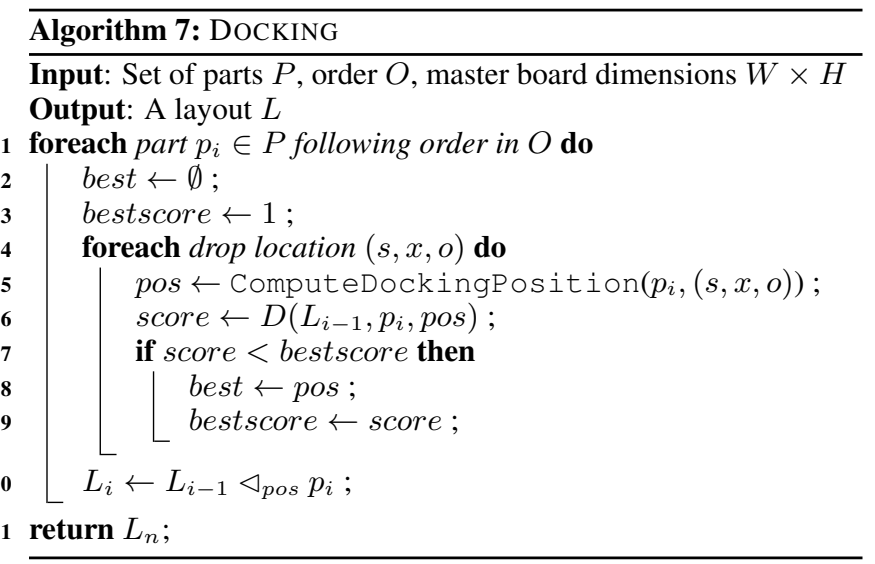

Docking criterion. The docking criterion considers wastage as the primary objective, where wastage is defined by the ratio of occupied area divided by the bounding rectangle area of the layout. We denote $W\left(L_{i}\right)$ the wastage of a layout including up to part $i$. It is obtained as $W\left(L_{i}\right)=\frac{\sum_{k=0}^{i} A\left(p_{k}\right)}{A\left(\operatorname{box}\left(L_{i}\right)\right)}$ where $A$ measures area and $\operatorname{box}(L)$ is the bounding rectangle of the layout. $W$ is therefore the ratio between the area of the parts and the area of the bounding rectangle. 
(a) Parrot shelf

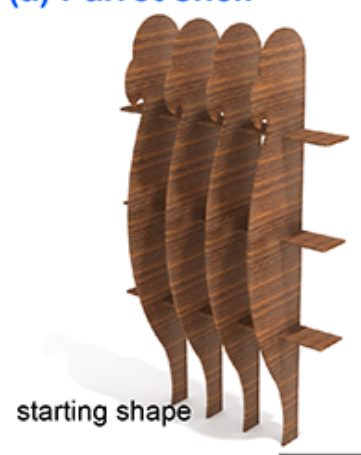

(b) Low chair

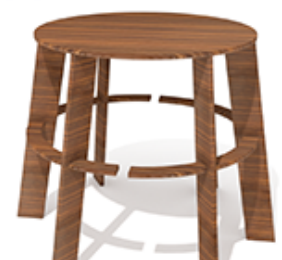

starting shape

(c) Ying chair

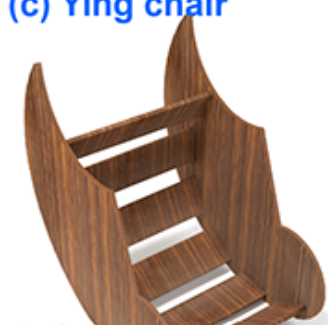

starting

shape input layout
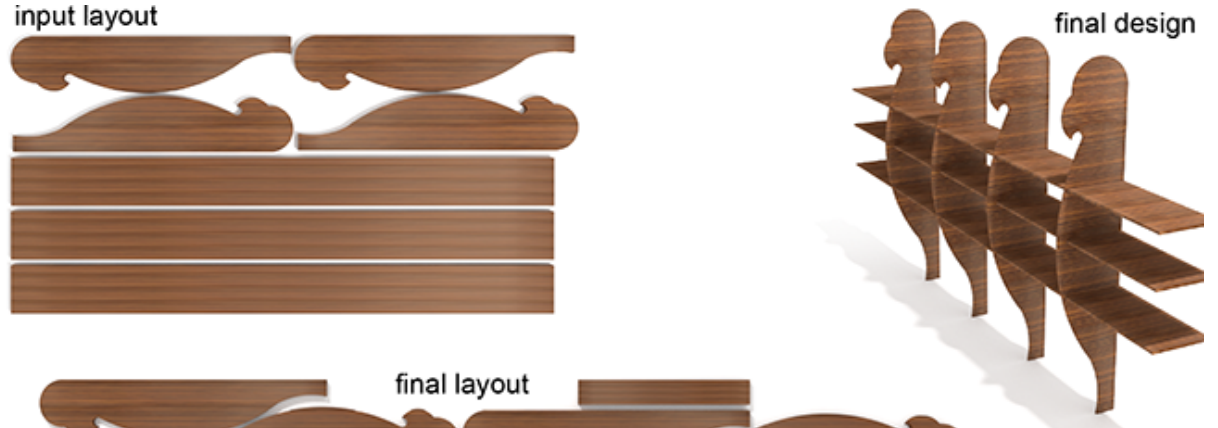

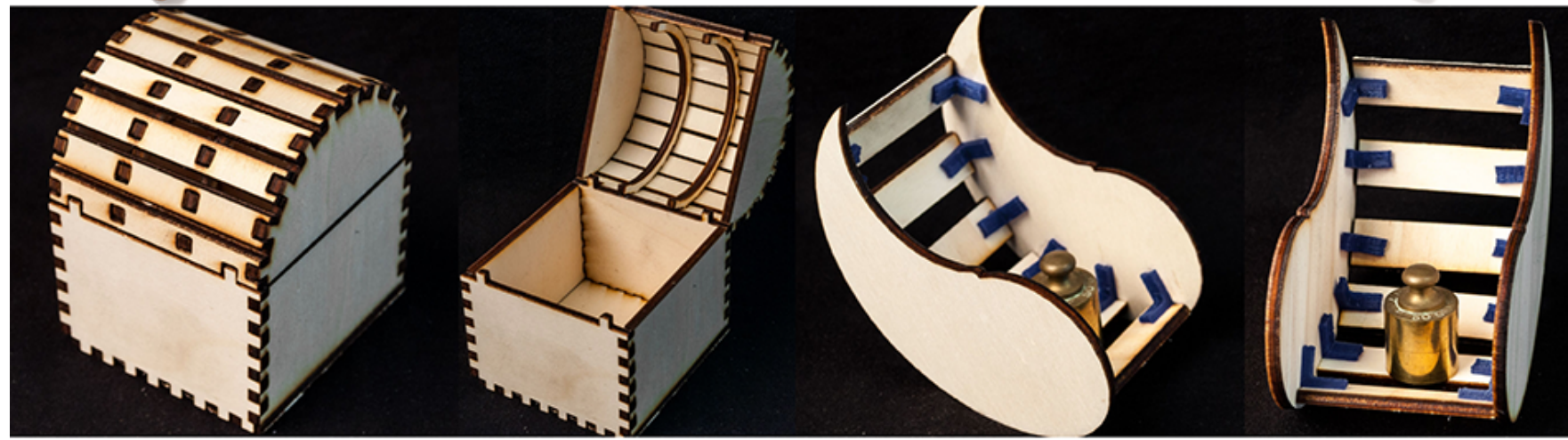

(d) Chest

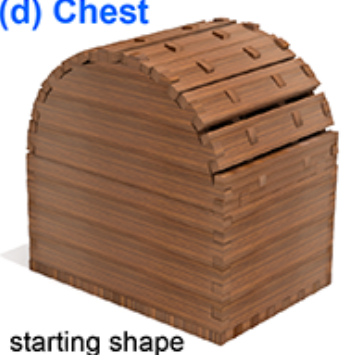

starting shape

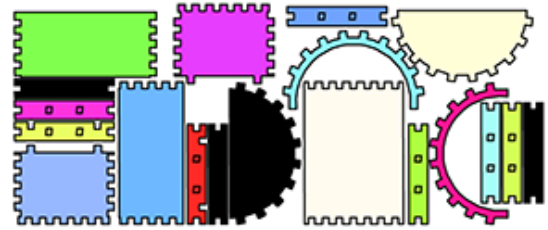

input layout

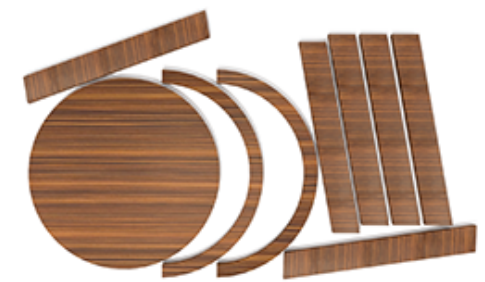

input layout

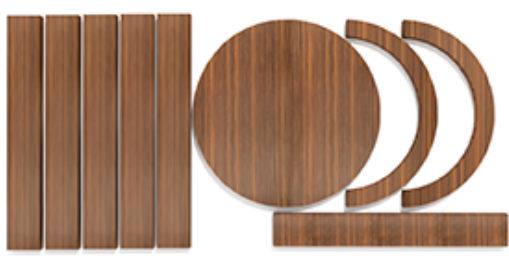

final layout
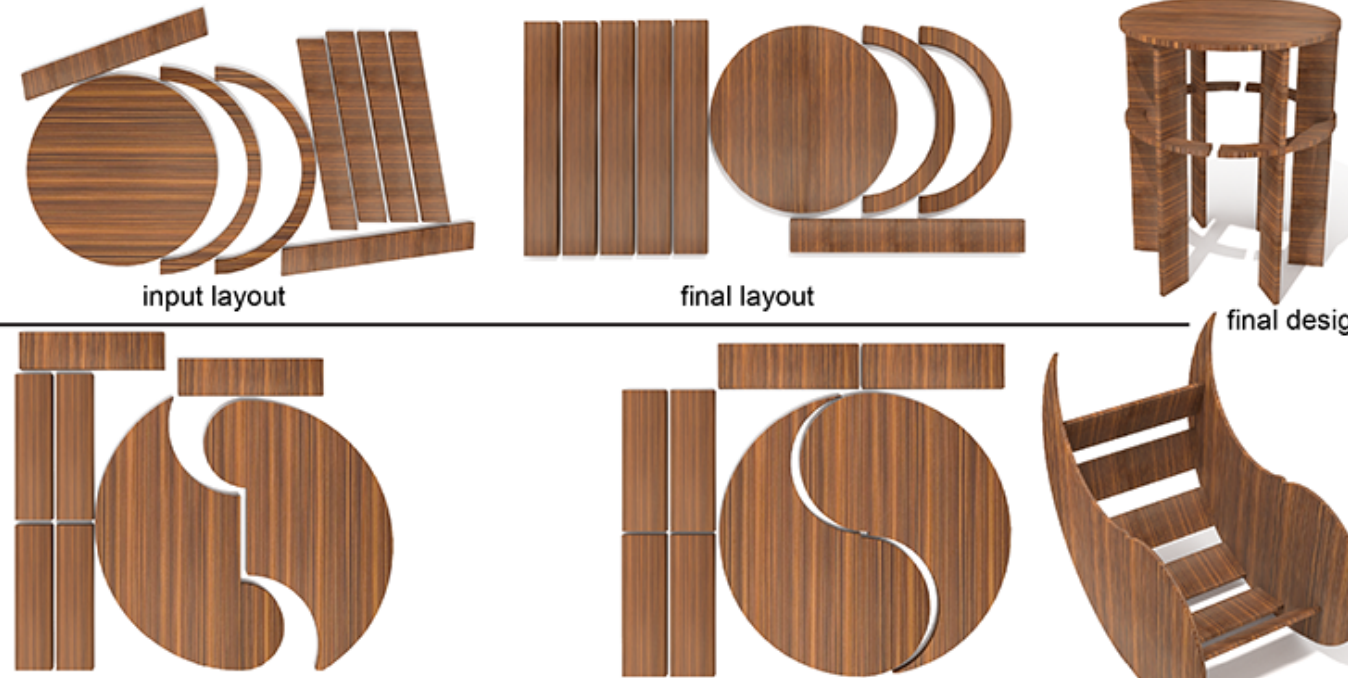

input layout
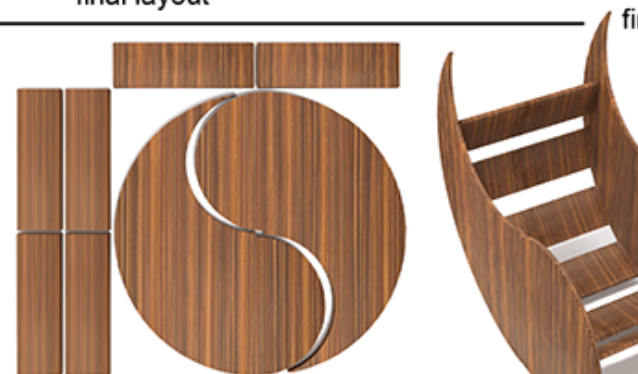

final design

Figure 7: Designs created using our system. Each design is shown with initial shape, starting layout, optimized layout, and final design. 
However, as the algorithm heuristically docks parts in sequence it cannot foresee that some spaces will be definitely enclosed. In particular, for newly inserted concave parts there are often multiple orientations of the part resulting in the same wastage: if the concavity remains empty there is no preferred choice. However, some choices are indeed better than others. If the concavity faces an already placed object, then further docking within the concavity will never be possible. This is illustrated in Figure 8, left.

We therefore propose a second criterion that discourages these bad choices. The idea is to estimate the space that will be definitely enclosed when a part is added to the current layout. This is done efficiently by considering the enclosed space between the heightfield of the current layout and the height-field of the added part, along both horizontal and vertical directions.

Let $H^{r}(L)$ (respectively $H^{t}$ ) be the right (respectively top) heightfield of layout $L$ and $A\left(H^{r}(L)\right)$ the area below it. The enclosed area is then defined as:

$$
\begin{aligned}
& E\left(L_{i-1}, p_{i}, \text { pos }\right)= \\
& \sum_{s \in\{r, t\}} \max \left(0, A\left(H^{s}\left(L_{i-1} \triangleleft_{\text {pos }} p_{i}\right)\right)-A\left(H^{s}\left(L_{i-1}\right)\right)-A\left(p_{i}\right)\right)
\end{aligned}
$$

with $A\left(p_{i}\right)$ the area of part $p_{i}$. Note the max that clamps negative values: this is due to cases where the part nests in a concavity below the height-field of the other direction.

The enclosed space is used as a tie-breaker when docking positions produce the same wastage values; therefore $D\left(L_{i-1}, p_{i}\right.$, pos $)$ returns the vector $\left(W\left(L_{i-1} \triangleleft_{\text {pos }} p_{i}\right), E\left(L_{i-1}, p_{i}, p o s\right)\right)$. The effect of the enclosed area criterion is shown in Figure 8.
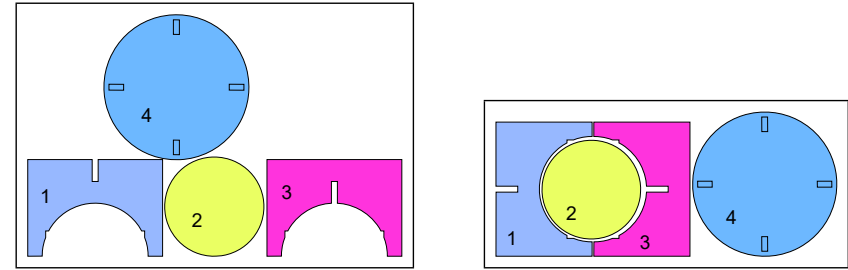

Figure 8: Two layouts obtained with the same docking order. Left: Without taking enclosed area into account the first part is placed with the concavity against the bottom packing border. This prevents the second part to nest within and cascades into a series of poor placements. Right: Taking into account enclosed areas results in a placement of the first part that allows nesting of the second part and produces a layout with lower wastage.
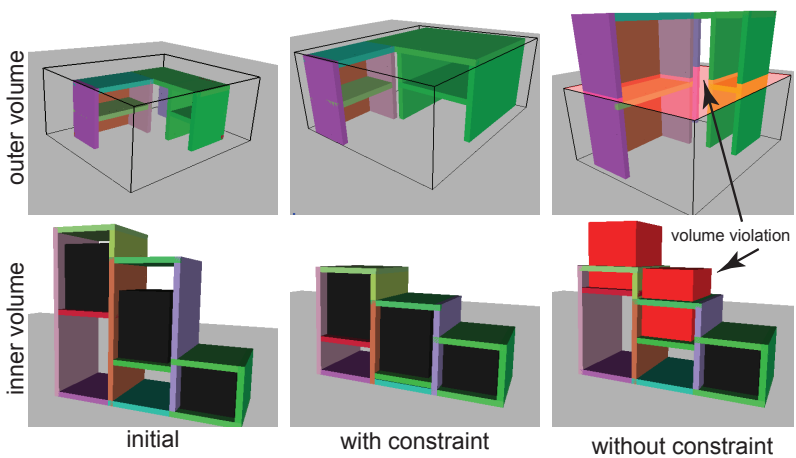

Figure 9: We show effects of designing with (middle column) or without (right column) the respective constraints activated.

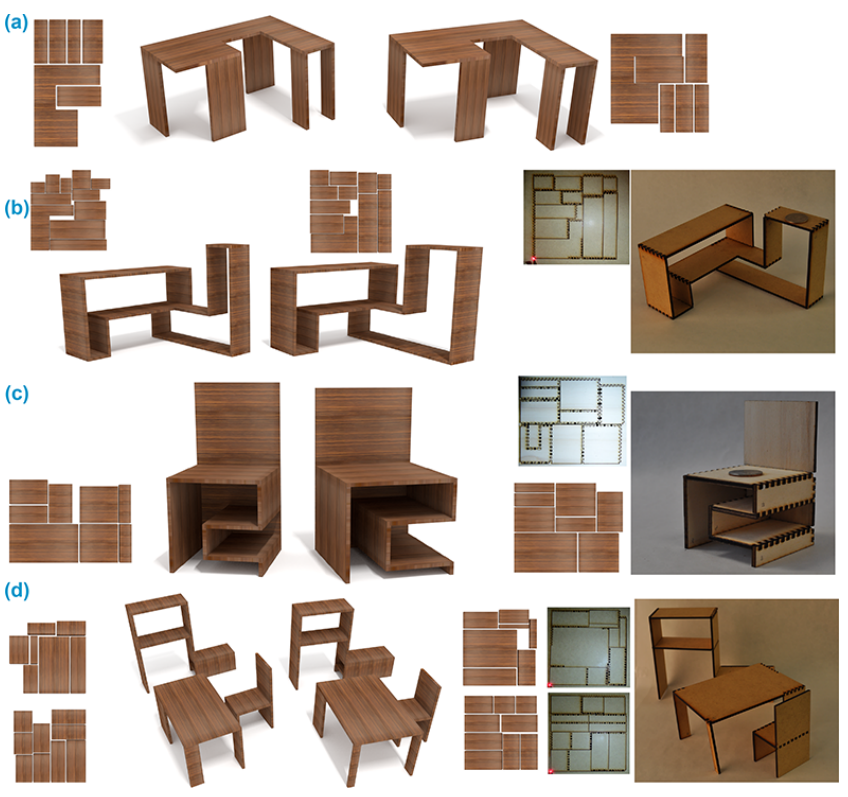

Figure 10: Various material-driven design and fabrication examples. In each row, we show initial design (with material space layout inset), optimized design result (with material space layout inset), along with final cutout assembled model. Note that the design changes are often subtle, but still leads to significant improvement in material usage.

\section{Results}

We used our system for various design explorations. As the complexity of the designs grows beyond 4-6 planks, the utility of the system quickly becomes apparent. Note that the design constraints (see Figure 9), by coupling different object parts, make the optimization challenging by preventing independent adaptation of part sizes. By off-loading material usage considerations to the system, the user can focus on the design. Note that even when changes to the design are visually subtle, material utilization often increases significantly.

Design examples. We used our system to design and fabricate a range of examples comprising rectangular and/or curved parts. We fabricated fullscale and miniature models of designed furniture. Models were made from MDF of $3 \mathrm{~mm}$ thickness and MDF of $30 \mathrm{~mm}$ thickness. The designs are easy to manufacture in batches since after design layout optimization they typically fit master boards completely: there is no need to attempt to reuse leftover pieces of wood, and switching boards requires little clean up.

We directly output the cutting plan for the laser cutter (or CNC machine) from the design layout, adding connectors for planks sharing an edge, if needed. These are conveniently detected since planks exactly overlap on edges in the 3D design. The connectors are either finger joints, which are both strong after gluing and easy to assemble; cross connectors for interleaved planks, or dowel-jointed for thicker materials ( $20 \mathrm{~mm}$ and $30 \mathrm{~mm}$ thickness).

Figures 7 and 10 show various results. Table 1 gives an overview of the complexity of each model, and the gains obtained by the layout optimizer. The system performs at interactive rates on a laptop taking from a few seconds to 3-4 minutes for the larger examples. Note that speed depends on how many exploration threads are pursued.

Figures 1 and 7 show results for objects with curved parts. Figure 3 shows some intermediate shapes as the design evolves for the 
Table 1: Statistics for cut design showing the number of planks, number of constraints, material wastage ratio before and after the design suggestions/optimization.

\begin{tabular}{l|c|c|c|c} 
& \#planks & \#constraints & ratio before & ratio after \\
\hline Figure 1 & 4 & 21 & 0.22 & 0.11 \\
Figure 7a & 7 & 33 & 0.34 & 0.08 \\
Figure 7b & 9 & N/A & 0.34 & 0.20 \\
Figure 7c & 8 & N/A & 0.24 & 0.17 \\
Figure 7d & 16 & N/A & 0.21 & 0.14 \\
Figure 10a & 6 & 22 & 0.15 & 0.04 \\
Figure 10b & 11 & 41 & 0.15 & 0.03 \\
Figure 10c & 8 & 13 & 0.26 & 0.03 \\
Figure 10d & 16 & 29 & 0.11 & 0.02
\end{tabular}

coffee-table (Figure 1) and the low-chair (Figures 7-top) examples. Figure 11 shows alternate designs discovered by the algorithm for the Parrot shelf. While they have slightly lower usage they offer interesting variations that the user might prefer.

Figures 1 was fabricated using a CNC machine. The optimized design achieved nearly $90 \%$ material usage, although one can achieve null wastage by deciding to pick a rectangular top - a decision that can be made after layout optimization as this opportunity is revealed. An allowable range was specified for the height and the bases were marked as symmetric as input design constraints. In the case of the parrot-shelf (Figure 7a), the user indicated minimum and maximum range for the horizontal shelves along with desired range for the shelf heights.

As described, parameteric designs are easily supported and optimized for in our framework. Figures $7 \mathrm{~b}$-d show three such examples. In each case, additional constraints were provided to keep the objects within a given volume. The parts of the objects are all tightly coupled making these challenging examples to optimize for.

Figure 10a shows a L-shaped work table. The user specified a target height for the design and a maximum work volume. Note that the legs of the table were also constrained to not change more than $25 \%$ of original dimensions to prevent unwanted design changes. Figure 10b shows a coupled shelf and table design where height of shelves and tabletop were similarly constrained. Figure 10c shows a

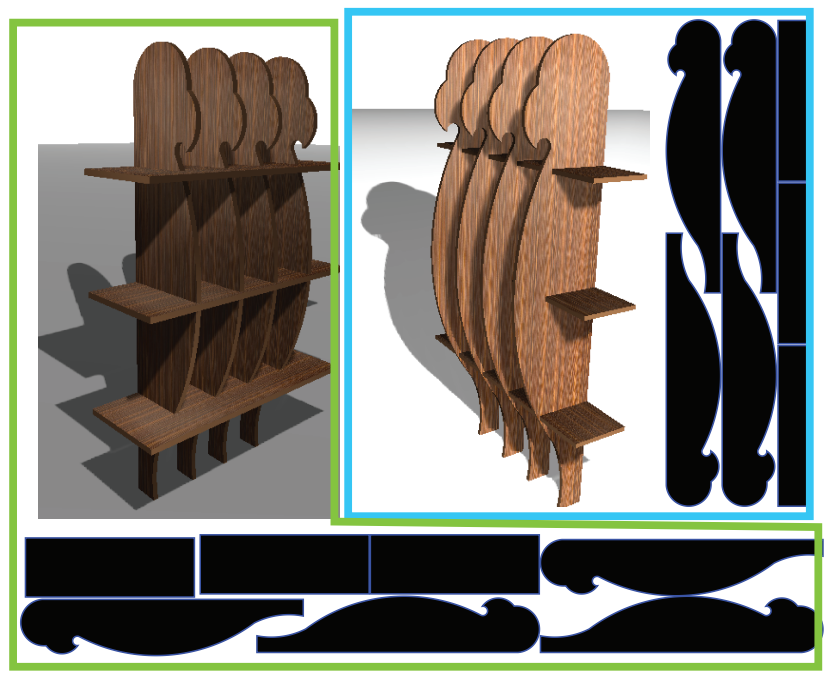

Figure 11: Two different design suggestions (green has ratio 0.86, blue has ratio 0.85) for the parrot-shelf. Original design with another design suggestion is shown in Figure 7.
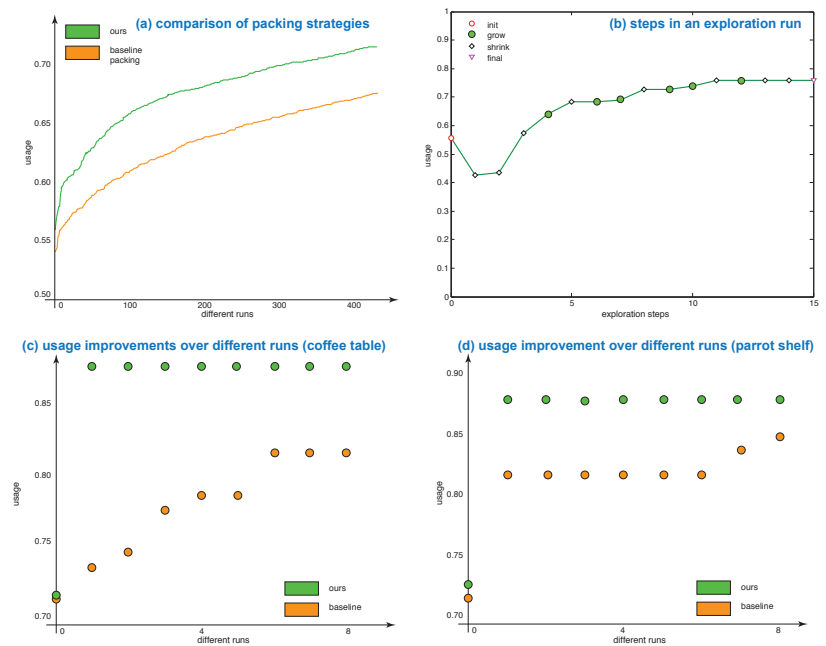

Figure 12: Comparison of our algorithm against baseline alternatives. Higher is better. Please refer to the text for details.

stylized chair, where both the chair seat height and chair width were constrained not to change beyond a margin. Figure 10d shows multiple designs covering 2 master boards. The second master board is used as an overflow when docking can no longer fit a part in the first. The layouts are slid independently.

Comparison. We now evaluate the relative importance of the key algorithm steps. Figure 12a shows the importance of the docking criteria introduced in Section 5.2. We ran 500 random runs of our proposed packing algorithm with ('ours') and without ('baseline') the docking criteria on the coffee-table example. We sort the runs based on resultant usage (no shape optimization is performed here) and plot the two conditions. The docking criteria consistently resulted in $10-15 \%$ better usage.

Figure 12b shows usage improvement over one exploration run on the coffee-table sequence. The legend explains which step (grow, shrink, etc.) is being performed. While this is the result from a single thread, many similar threads are simultaneously explored. The few best results are then presented to the user as suggestions.

Figure 12c-d compare the importance of analyzing the material space layout to decide which plank to change and how. As baseline, we selected planks at random and perform either a grow or shrink sequence with equal probability. Note that our method consistently outperforms the alternative approach.

Design sessions. We asked second year art students (6 subjects) from a design college to try our system. Figures 10b-d show a selection of their designs. These particular students had performed a very similar task as part of their first year assignment - 'design furniture of your choice making best use of the provided piece of MDF board.' Hence, they were very aware of the implicit link between design and material usage. Previously, they had used commercial 3D modeling tool (Rhinoceros, Solidworks, Sketchup Pro) for designing and mainly Illustrator for manually laying out the designs. They recalled the frustration of having to switch between the different 2D-3D design representations. First, the students sketched design concepts before using our system. Then, they used the exploration interface on their designs to reduce wastage. Note that visually the initial sketch and final design can look similar, despite the increase in material utilization, which is desirable in terms of preserving the original design.

Overall, the feedback was positive. They appreciated being able to 
easily move between $2 \mathrm{D} \leftrightarrow 3 \mathrm{D}$, and not having to explicitly worry about material utilization. They appreciated the suggestions, instead of previous attempts using trial-and-error iterations between various softwares to reduce material wastage.

Limitations. Currently, the algorithm can only make topological changes only for parameteric models. This will be an interesting future direction to pursue for constrained models. Our docking approach cannot nest parts into holes of other parts, a more advanced algorithm would be required. A more material-induced restriction arises when the starting layout does not leave much space to optimize over. This effectively means that the degree of freedom for the design is low. Adding more planks does reduce this problem (by providing additional freedom). However, beyond 25-30 planks, the exploration of the shape space becomes slow as there are too many paths to explore. One option is to limit exploration to only a subset of planks at a time, but then again, very desirable design configurations may be missed.

\section{Conclusions and Future Work}

We investigated how design constraints and material usage can be linked together towards form finding. Our system dynamically discovers and adapts to constraints arising due to current material usage, and computationally generates design variations to reduce material wastage. By dynamically analyzing 2D material space layouts, we determine which and how to modify object parts, while using design constraints to determine how the proposed changes can be realized. This interplay results in a tight coupling between 3D design and 2D material usage and reveals information that usually remains largely invisible to the designers, and hence difficult to account for. We used our system to generate a variety of shapes and demonstrated wastage reduction by $10 \%$ to $15 \%$.

Currently, we do not consider the stability of the produced furniture nor the durability of the joints. This could be integrated as dynamic constraints following previous work on structural reinforcement [Stava et al. 2012] and shape balancing [Prévost et al. 2013]. Another important future direction is to generalize the framework to handle other types of laser cut materials, e.g., plastic plates that can be easily cut and more interestingly bend to have freeform shapes. Note that the packing problem will still be in 2D for such developable pieces. This can help produce interesting freeform shapes, while still making efficient use of materials.

\section{Acknowledgements}

The work was supported in part by ERC ShapeForge (StG-2012307877) and ERC SmartGeometry (StG-2013-335373).

\section{References}

Bächer, M., Bickel, B., James, D. L., And Pfister, H. 2012. Fabricating articulated characters from skinned meshes. ACM Trans. Graph. (Proc. SIGGRAPH) 31, 4.

BFM. 2003. Wood waste recycling in furniture manufacturing. Tech. rep., British Furniture Manufacturer.

BoKeloh, M., Wand, M., Seidel, H.-P., And Koltun, V. 2012. An algebraic model for parameterized shape editing. ACM SIGGRAPH 31, 4, 78:1-78:10.

Brennan, G., Brown, J., Docherty, M., and Tullett, B. 2006. FlatPack/PlaskaPaczka. The Caseroom Press.
Brüderlin, B., AND Roller, D., Eds. 1998. Geometric Constraint Solving and Applications, vol. VIII. Springer.

Calì, J., Calian, D., Amati, C., Kleinberger, R., Steed, A., KAUTZ, J., AND WEYRICH, T. 2012. 3d-printing of nonassembly, articulated models. 130:1-130:8.

Chen, S. S., Donoho, D. L., And Saunders, M. A. 2001. Atomic decomposition by basis pursuit. SIAM Rev. 43, 1, 129159.

Chen, X., Zhang, H., Lin, J., Hu, R., Lu, L., Huang, Q., Benes, B., COHEn-Or, D., AND CHEN, B. 2015. Dapper: Decompose-and-pack for 3d printing. ACM Trans. Graph. 34, 6 (Oct.), 213:1-213:12.

Cignoni, P., Pietroni, N., Malomo, L., And Scopigno, R. 2014. Field-aligned mesh joinery. ACM TOG 33, 1 (January).

Coros, S., Thomaszewski, B., Noris, G., Sueda, S., ForBerG, M., Sumner, R. W., MATusiK, W., AND Bickel, B. 2013. Computational design of mechanical characters. $A C M$ SIGGRAPH 32, 4, 83:1-83:12.

DAIAN, G., AND OZARSKA, B. 2009. Wood waste management practices and strategies to increase sustainability standards in the australian wooden furniture manufacturing sector. Journal of Cleaner Production 17, 17, 1594?-1602.

Dumas, J., Hergel, J., AND Lefebvre, S. 2014. Bridging the gap: Automated steady scaffoldings for 3d printing. ACM Trans. Graph. 33, 4 (July), 98:1-98:10.

Fu, C.-W., Song, P., Yan, X., Yang, L. W., Jayaraman, P. K., AND COHEN-OR, D. 2015. Computational interlocking furniture assembly. ACM Trans. Graph. 34, 4 (July), 91:1-91:11.

Gal, R., Sorkine, O., Mitra, N. J., And Cohen-Or, D. 2009. iwires: An analyze-and-edit approach to shape manipulation. ACM Transactions on Graphics (Siggraph) 28, 3, \#33, $1-10$.

Guennebaud, G., JACOB, B., ET AL., 2010. Eigen v3. http://eigen.tuxfamily.org.

HabBecke, M., And KobBelt, L. 2012. Linear analysis of nonlinear constraints for interactive geometric modeling. $C G F$ $31,2,641-650$.

Hildebrand, K., Bickel, B., And AleXA, M. 2012. crdbrd : Shape fabrication by sliding planar slices. CGF Eurographics 31,2 .

Hu, K., Jin, S., AND Wang, C. C. 2015. Support slimming for single material based additive manufacturing. Computer-Aided Design 65, 1 - 10 .

JYLÄNKI, J. 2010. A thousand ways to pack the bin-a practical approach to two-dimensional rectangle bin packing. Retrieved from http://clb.demon.fi/files/RectangleBinPack.pdf.

LI, X.-Y., Shen, C.-H., Huang, S.-S., Ju, T., And Hu, S.-M. 2010. Popup: automatic paper architectures from $3 \mathrm{~d}$ models. ACM SIGGRAPH 29, 4, 111:1-9.

LI, X.-Y., JU, T., GU, Y., AND HU, S.-M. 2011. A geometric study of v-style pop-ups: Theories and algorithms. ACM Transactions on Graphics 30, 4, 98:1-10.

Lu, L., Sharf, A., Zhao, H., Wei, Y., Fan, Q., Chen, X., Savoye, Y., Tu, C., Cohen-Or, D., And Chen, B. 2014. Build-to-last: Strength to weight 3d printed objects. ACM Trans. Graph. 33, 4 (July), 97:1-97:10. 
Luo, L., Baran, I., Rusinkiewicz, S., And Matusik, W. 2012. Chopper: Partitioning models into 3D-printable parts. ACM SIGGRAPH Asia 31, 6.

McCrae, J., Singh, K., And Mitra, N. J. 2011. Slices: A shape-proxy based on planar sections. ACM Transactions on Graphics 30, 6, 168:1-168:12.

Mueller, S., Lopes, P., AND BAudisch, P. 2012. Interactive construction: Interactive fabrication of functional mechanical devices. In Proc. UIST, ACM, New York, NY, USA, 599606.

Prévost, R., Whiting, E., Lefebvre, S., And SorkineHoRnung, O. 2013. Make it stand: Balancing shapes for 3d fabrication. ACM Trans. Graph. 32, 4 (July), 81:1-81:10.

SaAkes, D., Cambazard, T., Mitani, J., And Igarashi, T. 2013. Paccam: Material capture and interactive $2 \mathrm{~d}$ packing for efficient material usage on cnc cutting machines. In Proc. UIST, ACM, New York, NY, USA, 441-446.

Schulz, A., Shamir, A., Levin, D. I. W., Sitthi-Amorn, P., AND MatusiK, W. 2014. Design and fabrication by example. ACM Trans. Graph. 33, 4 (July), 62:1-62:11.

SCHWARTZBURG, Y., AND PAULY, M. 2013. Fabrication-aware design with intersecting planar pieces. CGF Eurographics 32, 2, 317-326.

Shugrina, M., Shamir, A., And Matusik, W. 2015. Fab forms: Customizable objects for fabrication with validity and geometry caching. ACM Trans. Graph. 34, 4 (July), 100:1-100:12.

Stava, O., VAneK, J., Benes, B., CARr, N., AND MĚCh, R. 2012. Stress relief: Improving structural strength of $3 \mathrm{~d}$ printable objects. ACM Trans. Graph. 31, 4 (July), 48:1-48:11.

Talton, J. O., Gibson, D., Yang, L., Hanrahan, P., And Koltun, V. 2009. Exploratory modeling with collaborative design spaces. In ACM SIGGRAPH Asia, 167:1-167:10.

Umetani, N., Igarashi, T., And Mitra, N. J. 2012. Guided exploration of physically valid shapes for furniture design. $A C M$ SIGGRAPH 31, 4, 86:1-86:11.

Vanek, J., Galicia, J. A. G., Benes, B., Měch, R., Carr, N., Stava, O., AND Miller, G. S. 2014. Packmerger: A 3d print volume optimizer. Computer Graphics Forum 33, 6, $322-332$.

Wang, W., Wang, T. Y., Yang, Z., LiU, L., Tong, X., Tong, W., Deng, J., Chen, F., And LiU, X. 2013. Cost-effective printing of $3 \mathrm{~d}$ objects with skin-frame structures. ACM Trans. Graph. 32, 6 (Nov.), 177:1-177:10.

Xu, W., Wang, J., Yin, K., Zhou, K., van de Panne, M., CHEN, F., AND GUO, B. 2009. Joint-aware manipulation of deformable models. ACM Trans. Graph. 28, 3 (July), 35:1-35:9.

Xu, K., Zhang, H., Cohen-Or, D., And Chen, B. 2012. Fit and diverse: Set evolution for inspiring $3 \mathrm{~d}$ shape galleries. $A C M$ SIGGRAPH 31, 4, 57:1-10.

YAo, M., Chen, Z., LuO, L., WANG, R., AND WANG, H. 2015. Level-set-based partitioning and packing optimization of a printable model. ACM Trans. Graph. 34, 6 (Oct.), 214:1-214:11.

Zheng, Y., Fu, H., Cohen-Or, D., Au, O. K.-C., And TAI, C.-L. 2011. Component-wise controllers for structurepreserving shape manipulation. In Computer Graphics Forum (In Proc. of Eurographics 2011), vol. 30.
Zhou, Q., PAnetTa, J., AND Zorin, D. 2013. Worst-case structural analysis. ACM Trans. Graph. 32, 4 (July), 137:1-137:12. 